\title{
Enhancement of Rashba interaction in GaAs/AIGaAs quantum wells due to the incorporation of bismuth
}

\author{
R.A. Simmons, ${ }^{1}$ S.R. Jin, ${ }^{1}$ S.J. Sweeney, ${ }^{1}$ and S.K. Clowes ${ }^{1}$ \\ Advanced Technology Institute and Department of Physics, University of Surrey, Guildford, Surrey, \\ GU2 $7 X H$ U.K.
}

This paper reports on the predicted increase in the Rashba interaction due to the incorporation of Bi in GaAs/AlGaAs heterostructures. Band structure parameters obtained from the band anti-crossing theory have been used in combination with self-consistent Schrödinger-Poisson calculations and k.p models to determine the electron spin-splitting caused by structural inversion asymmetry and increased spin-orbit interaction. A near linear seven fold increase in the strength of the Rashba interaction is predicted for a $10 \%$ concentration of $\mathrm{Bi}$ in a $\mathrm{GaAsBi} / \mathrm{AlGaAs}$ quantum well heterostructure.

Over the past few years the growth of III-V bismide heterostructures has witnessed significant advances due to their potential for improved efficiency in photonic devices in the near infrared region. ${ }^{1-4}$ The origin of these potential efficiency gains is an increased spin-orbit band separation $\left(\Delta_{S O}\right)$ in the bismides which suppresses dominant non-radiative Auger recombination processes. ${ }^{5}$ This increase in the spin-orbit interaction offered by the bismides also has potential to be exploited in semiconductor spintronics. For n-type semiconductors to be able to operate at elevated temperatures with high electron spin modulation frequencies and nanoscale dimensions, it is necessary to increase the conduction band spin splitting $\left(\Delta E_{S I A}\right)$ due to structural inversion asymmetry. ${ }^{6}$

The narrowgap semiconductors InAs and InSb exhibit the strongest conduction spin-orbit coupling due to large atomic masses and low effective electron mass $\left(m^{*}\right)$ and have been much reported for this reason. ${ }^{7-10}$ The largest observed $\Delta E_{S I A}$ have been reported for asymmetric $\mathrm{InSb} / \mathrm{InAlSb}$ quantum well (QW) structures where the typical conduction band spin splitting is of the order of $3 \mathrm{meV} .{ }^{11}$ This has led to the demonstration of spin dependent electron transport in these structures at temperatures up to approximately $20 \mathrm{~K}$ and spin coherence lengths of up to $2 \mu \mathrm{m} .{ }^{12,13}$ Despite $T_{2}$ spin lifetimes of the order of a $\mathrm{ps}^{14,15}$ arising from spin precessional frequencies in the $\mathrm{THz}$ range, these observations are possible since Fermi velocities $\left(v_{F}\right)$ in these materials can be of the order of a $10^{6} \mathrm{~ms}^{-1}$. Counterintuitively, the fact that these materials exhibit long spin coherence lengths limits the ability to scale down device dimensions as the distance required to precess the electron spin can be measured in micrometres. New materials are therefore required that exhibit much higher conduction band spin splittings, which can be achieved with an increased spin-orbit interaction and a reduced bandgap $\left(E_{g}\right)$.

Incorporation of $\mathrm{Bi}$ into III-V materials may offer the solution due to the higher atomic mass of $\mathrm{Bi}$ which increases $\Delta_{S O}$ as well as reducing $E_{g}$ due to a resonant Bi level lying below the valence band maximum causing a considerable anti-crossing perturbation ${ }^{16}$ leading to an upward movement of the valence band edge. The

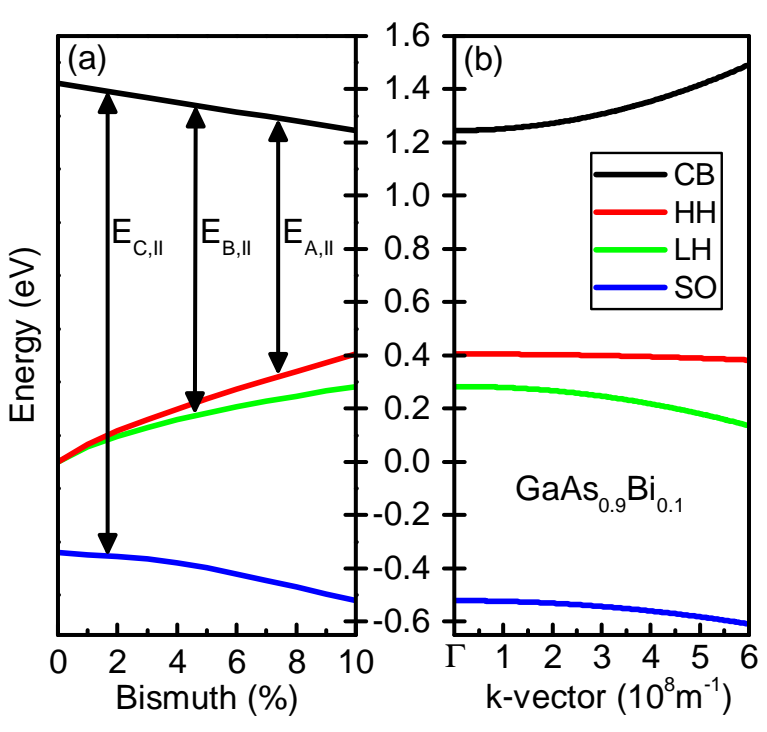

Figure 1. (a) Diagram showing the changing of the band structure of GaAs due to bismuth incorporation for $\mathrm{T}=300 \mathrm{~K}$. These are calculated from a parameterisation of a 12-band tight binding model. ${ }^{16}$ Note the breaking of the valence band degeneracy due to strain. The model parameters are indicated and defined as follows. $E_{A}=C B-H H, E_{B}=C B-L H$, and $E_{C}=C B-S O$. (b) A schematic of the bandstructure of $G a A s_{0.9} B i_{0.1}$. This diagram shows the weighted average ${ }^{17}$ of the band gaps at $\Gamma$, with a simple parabolic dispersion using the predicted effective mass, $m^{*}=0.0544$.

effects of these perturbations are illustrated in Fig. 1. Another predicted effect of bismuth incorporation is the reduction of conduction band effective mass, which leads directly to an increase in electron mobility and $\Delta E_{S I A}$.

The Rashba interaction originates from structural inversion asymmetries (SIA) arising from either an applied or an in-built electric field due to asymmetries in the heterostructure. This lifts the conduction band spin degeneracy with $\Delta E_{S I A}=2 \alpha_{R} k_{F}$ where $k_{F}$ is the Fermi crystal momentum and $\alpha_{R}$ is the Rashba parameter. ${ }^{6}$ In terms of the bulk material properties the value of 
$\alpha_{R}$ in relation to the electric field $F$ arising from SIA is $\alpha_{R} \approx \alpha_{0} e F$, where $\alpha_{0}$ is specific to the band structure of the material. While the effect of the average field is related to the total spin splitting, it is more fitting to consider the effect it has on the distribution of the wavefunction in a quantum well. While this linear relation is not strictly accurate it provides a good figure of merit when determining the material potential for spintronics, providing a measure of the ease with which an applied electric field can modulate $\Delta E_{S I A}$. It has been shown by k.p theory that increasing $\Delta_{S O}$ and reducing $E_{g}$ should lead to an enhancement in Rashba coefficient $\alpha_{0} .{ }^{18}$

It has recently been reported that calculations using $\Delta_{S O}$ and $E_{g}$ overestimate the electronic properties of $\mathrm{GaAs}_{1-x} \mathrm{Bi}_{x}$ due to mixed states in the valence band from alloy disorder ${ }^{19}$, as well as valence band anti-crossing effects. ${ }^{20}$ Therefore in this work we use parameterised values from the 12-band tight binding model of Broderick et $\mathrm{al}^{16}$ to take into account the effects of the mixed states, pseudomorphic strain, and band anti-crossing effects. A weighted average is taken for the band edges to account for the fractional $\Gamma$ character of the band edges. The validity of this technique has been previously demonstrated in studies of the anisotropic g factor in $\mathrm{GaAs}_{1-x} \mathrm{Bi}_{x}$ alloys. ${ }^{17}$ In this paper we will present the modeling of GaAs/AlGaAs modulation doped QW structures that have been used to calculate the increased strength electron spin splitting due to Bi incorporation. As the conduction electrons are in a bound state along the growth direction, the effective field across the well is therefore not directly contributing to spin splitting. It is through the electric field altering the penetration of the wavefunction at the well-barrier interface that has been shown to be the dominant term from the Hamiltonian ${ }^{21}$. Consequently we have used the model proposed by Pfeffer and Zawadski ${ }^{21}$ which is derived from a k.p Hamiltonian $(\hat{H} \Phi=\lambda \Phi)$ taking into account the three energy levels of the $\Gamma_{6}^{c}$ conduction band, the $\Gamma_{8}^{v}$ valence band and the $\Gamma_{7}^{v}$ spin-orbit spin off band. This Hamiltonian is condensed to a $2 \times 2$ eigenvalue problem for the two conduction band states by including an additional operator into the diagonal elements to take into account the valence band contributions. Ignoring contributions arising from bulk inversion asymmetry (BIA), the conduction band spin splitting is $\Delta E_{S I A}=2\left\langle\Phi\left|\hat{K}_{S I A}\right| \Phi\right\rangle$ where the $\hat{K}_{S I A}$ is the off-diagonal structural inversion asymmetry (SIA) term in $2 \times 2$ matrix which gives rise to the Rashba interaction. In addition we have modified this term from that used by Pfeffer and Zawadski to take into account the the presence of pseudomorphic strain ${ }^{17}$ which lifts the $\mathrm{LH}$ and $\mathrm{HH}$ degeneracy, such that

$$
\hat{K}_{S I A}=\frac{k_{\|} P_{0}^{2}}{2} \frac{\partial}{\partial z}\left[\left(\frac{1}{\tilde{E_{A}}}-\frac{1}{3 \tilde{E_{B}}}-\frac{2}{3 \tilde{E_{C}}}\right)\right]
$$

where $\tilde{E_{A}}=E_{A}+\lambda-V(z), \tilde{E_{B}}=E_{B}+\lambda-V(z)$, $\tilde{E_{C}}=E_{C}+\lambda-V(z), P_{0}{ }^{2}=\hbar^{2} E_{p} / 2 m_{0}$ and $k_{\|}$is the electron momentum in the plane of the quantum well.
By averaging the $\hat{K}_{S I A}$ term taken over $\Phi(z)$, and taking into account the offsets in $E_{C B}, E_{H H}, E_{L H}$ and $E_{S O}$ at $z=0$ and $z=a$, the conduction band spin-splitting is

$$
\begin{array}{r}
\Delta E_{S I A}=\frac{k_{\|} \hbar^{2} E_{p}}{2 m_{0}}\left[\left\langle\Phi\left|\frac{-\partial V}{\partial z} D_{i}\right| \Phi\right\rangle\right. \\
\left.+\Phi^{2}(0) C_{0}-\Phi^{2}(a) C_{a}\right]
\end{array}
$$

where

$$
\begin{gathered}
D_{i}=\frac{1}{{\tilde{E_{A}}}^{2}}-\frac{1}{3{\tilde{E_{B}}}^{2}}-\frac{2}{3{\tilde{E_{C}}}^{2}} \\
C_{0}=\frac{1}{\tilde{E}_{A, I I 0}}-\frac{1}{3 \tilde{E}_{B, I I 0}}-\frac{2}{3 \tilde{E}_{C, I I 0}}-\frac{2}{3 \tilde{E}_{A, I 0}}+\frac{2}{3 \tilde{E}_{C, I 0}}
\end{gathered}
$$

and

$$
C_{a}=\frac{1}{\tilde{E}_{A, I I a}}-\frac{1}{3 \tilde{E}_{B, I I a}}-\frac{2}{3 \tilde{E}_{C, I I a}}-\frac{2}{3 \tilde{E}_{A, I a}}+\frac{2}{3 \tilde{E}_{C, I a}}
$$

where $\tilde{E}_{A, I I 0}=E_{A, I I}+\lambda, \tilde{E}_{B, I I 0}=E_{B, I I}+\lambda, \tilde{E}_{C, I I 0}=$ $E_{C, I I}+\lambda, \tilde{E}_{A, I 0}=E_{A, I}+\lambda-V_{B}, \tilde{E}_{C, I 0}=E_{C, I}+\lambda-V_{B}$, $\tilde{E}_{A, I I a}=E_{A, I I}+\lambda-V(a), \tilde{E}_{B, I I a}=E_{B, I I}+\lambda-V(a)$, $\tilde{E}_{C, I I a}=E_{C, I I}+\lambda-V(a), \tilde{E}_{A, I a}=E_{A, I}+\lambda-V_{B}-V(a)$, $\tilde{E}_{C, I a}=E_{C, I}+\lambda-V_{B}-V(a), V$ is potential of the $\Gamma_{6}^{c}$ band and in this study we used the value for bulk $\mathrm{GaAs}^{22}$ of $28.8 \mathrm{eV}$ for $E_{p}$. The first term in Eq. 2 excludes the contributions of the potential steps at the interfaces between regions I and II, as shown in Fig. 2. The effect of these potential jumps at $z=0$ and $z=a$ are incorporated in the second and third terms in Eq. 2, respectively. $\Phi(0)$ and $\Phi(a)$ are the amplitudes of the envelope function at $z=0$ and $z=a$, which been determined using the self-consistent solution of $\hat{H} \Phi=\lambda \Phi$ using the NextNano 1D Schrödinger-Poisson solver.

For this study we have investigated the effect of increasing the SIA interaction in modulation doped GaAsBi/AlGaAs QW structures by varying the modulation doping and $\mathrm{Bi}$ concentration. The simulated QWs consist of a doping layer $18 \mathrm{~nm}$ above an $18 \mathrm{~nm}$ $\mathrm{GaAs}_{1-x} \mathrm{Bi}_{x}$ QW (region II), as shown in Fig. 2. The barrier material (region I) was chosen to be $\mathrm{Al}_{0.3} \mathrm{Ga}_{0.7} \mathrm{As}$ as it has a type I band offset relative to GaAs and is also close to lattice matched for a wide range of aluminium concentrations. The band parameters shown in Fig. 1 were used for $E_{A, I I}, E_{B, I I}$ and $E_{C, I I}$ and for region I we used $E_{A, I}=E_{B, I}=1.833 \mathrm{eV}$ and $E_{C, I}=2.155 \mathrm{eV}$. The well dimensions were chosen for consistency with previous calculations, allowing for the comparison with Pfeffer and Zawadzki's ${ }^{21}$ calculations of the experimental results of Das et $\mathrm{al}^{23}$. The calculated spin splitting for three QWs with $\delta$-doping densities $n$ of $1.0 \times 10^{15}, 1.5 \times 10^{15}$ and $2.0 \times 10^{15} \mathrm{~cm}^{-2}$ are shown in Fig. 3(a). As expected an increase in $\Delta E_{S I A}$ is observed with increasing structural asymmetry and all doping concentrations show an 


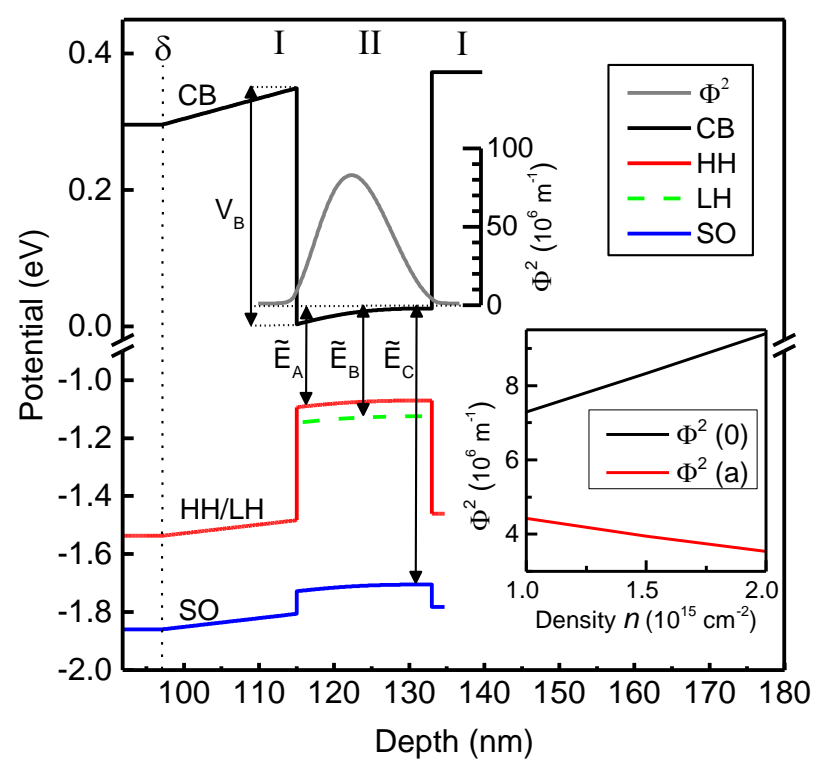

Figure 2. An example of the potential profile of the modulation doped quantum wells used in this study. This particular well is an $18 \mathrm{~nm}$ wide $\mathrm{Al}_{0.3} \mathrm{Ga}_{0.7} \mathrm{As} / \mathrm{GaAs}_{0.95} \mathrm{Bi}_{0.05} \mathrm{QW}$ with a $\delta$-doped layer $18 \mathrm{~nm}$ above the QW and a density of $2.0 \times 10^{15} \mathrm{~cm}^{-2}$. Here $\lambda$ is the eigen energy, and $V_{B}$ and $V(a)$ denote the potential of the barrier and the offset potential due to modulation doping respectively. Also shown are the parameters $\tilde{E}_{A}, \tilde{E}_{B}$, and $\tilde{E}_{C}$ which are defined in the text. Inset shows the change in expectation value at top $\left(\Phi(0)^{2}\right)$ and bottom $\left(\Phi(a)^{2}\right)$ barrier as a function of modulation doping density.

approximate seven-fold increase in $\Delta E_{S I A}$ by incorporating $10 \% \mathrm{Bi}$. In order to extract a value for $\alpha_{0}$ the effective field due to the doping layer was calculated by applying an external field to compensate the potential. The field at which symmetry was restored to the wavefunction was determined to be equal to the field due to the doping layer. For a $\mathrm{Al}_{0.3} \mathrm{Ga}_{0.7} \mathrm{As} / \mathrm{GaAs} \mathrm{QW}$ with no $\mathrm{Bi}$ incorporation $F=7.03 \times 10^{5}, 1.06 \times 10^{6}$ and $1.41 \times 10^{6} \mathrm{Vm}^{-1}$ for $n=1.0 \times 10^{15}, 1.5 \times 10^{15}$ and $2.0 \times 10^{15} \mathrm{~cm}^{-2}$, respectively. Bi content has very little effect on these values, contributing to a slight decrease in the value of $F$ by less than $1 \%$ for concentrations up to $10 \%$. Fig. 3(b) shows $\alpha_{0}$ as a function of $\mathrm{Bi}$ concentration compensated for the differences in $F$ for the three doping concentrations using the relation $\alpha_{R} \approx \alpha_{0} e F$. It can be seen that the value of $\alpha_{0}$ is nearly independent of the structure of the QW and exhibits a near linear increase with Bi concentration, which is approximately seven times higher than that of the GaAs case for $10 \% \mathrm{Bi}$. As has already been stated the net field should be zero for the bound electron state, but as our results predict, the effective field is strongly coupled to the magnitude of $\alpha_{R}$ due to the redistribution of the wavefunction and therefore an approximation to linear dependency on the applied electric field is rea-

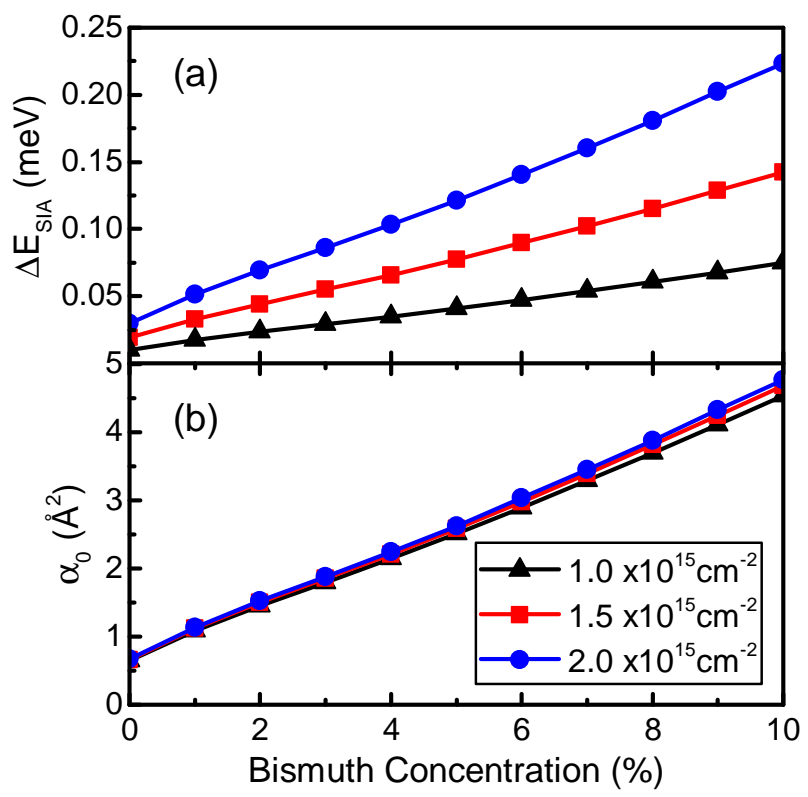

Figure 3. (a) Predicted strength of the conduction band Rashba spin splitting energy $\Delta E_{S I A}$ for different modulation doping concentrations. We observe a greater than seven-fold increase in energy splitting for $10 \% \mathrm{Bi}$, irrespective of doping density. (b) Rashba parameter $\alpha_{0}$ as a function of $\mathrm{Bi}$ incorporation in $18 \mathrm{~nm}$ wide $\mathrm{Al}_{0.3} \mathrm{Ga}_{0.7} \mathrm{As} / \mathrm{GaAs}_{1-x} \mathrm{Bi}_{x} \mathrm{QW}$. Here we see the increase of the material parameter independent of the structure.

sonable. Nevertheless, the importance of our results is that we predict that $\mathrm{Bi}$ incorporation has a significant effect on the magnitude of the conduction band spinsplitting and, more importantly, the ease by which this splitting can be modulated by an applied electric field.

While these results indicate that a significant increase in the Rashba interaction in GaAs based materials can be achieved with the incorporation of $\mathrm{Bi}$, the magnitude of $\Delta E_{S I A}$ that we predict remains too small for practical spintronics. The precession frequency of the conduction electron spin is $\omega_{p}=\Delta E_{S I A} / \hbar$ which gives the distance required to achieve a $\pi$ spin rotation as

$$
d_{\pi}=\frac{\pi \hbar^{2}}{2 \alpha_{0} e F m^{*}} .
$$

Note that this term is independent of $k_{\|}$as both $v_{f}$ and $\omega_{p}$ are proportional to $k_{\|}$. From our calculation of a GaAs QW with $n=2.0 \times 10^{15} \mathrm{~cm}^{-2}$ and $\Delta E_{S I A}=0.025 \mathrm{meV}$, the precession frequency would be $39 \mathrm{GHz}$ resulting in device dimensions of the order of millimeters necessary to allow a $\pi$ rotation of the electron spin. If we compare this to the $\mathrm{GaAs}_{0.9} \mathrm{Bi}_{0.1}$ case, where $\Delta E_{S I A}=0.22 \mathrm{meV}$, we increase the precession frequency to $0.3 \mathrm{THz}$, and therefore obtain $d_{\pi}$ on the order of $\geq 250 \mu \mathrm{m}$. Whilst this is clearly still too large for practical applications, the same principles of Rashba coefficient enhancement could apply to narrow gap materials such as InSb and InAs. A typical 
length for $d_{\pi}$ in InSb is of the order of a micron, and as such the large predicted enhancement in the Rashba parameter due to bismuth incorporation could potentially bring this length into the nanometer range.

Following the experimental verification of the findings reported in this study it will be necessary to look to the indium based ternary alloys InSbBi and InAsBi, which have an increased spin-orbit interaction and reduced bandgap ${ }^{24-27}$. Due to their strong potential for long wavelength $(8-14 \mu m)$ detection there has been significant interest in these alloys. Whilst growth of this material faces similar challenges to those faced by GaAsBi, recent reports have been very encouraging. ${ }^{27}$ When high quality indium based materials are available and if a similar Rashba enhancement is observed then there is great potential for these materials to be exploited for use in spintronics.

The authors gratefully acknowledge EPSRC (UK) for grant ref EP/H005587/1 and for a studentship for RS. The authors would also like to thank Z. L. Bushell, C. A. Broderick, and B. N. Murdin for helpful discussions.

\section{REFERENCES}

${ }^{1}$ S. J. Sweeney and S. R. Jin, J. Appl. Phys. 113, 043110 (2013). ${ }^{2}$ P. Ludewig, Z. L. Bushell, L. Nattermann, N. Knaub, W. Stolz, and K. o. s. Volz, J. Cryst. Growth 396, 95 (2014).

${ }^{3}$ I. P. Marko, P. Ludewig, Z. L. Bushell, S. R. Jin, K. Hild, Z. Batool, S. Reinhard, L. Nattermann, W. Stolz, K. Volz, and S. J. Sweeney, J. Phys. D: Appl. Phys 47, 345103 (2014).

${ }^{4}$ R. D. Richards, F. Bastiman, C. J. Hunter, D. F. Mendes, A. R. Mohmad, J. S. Roberts, and J. P. R. David, J. Cryst. Growth 390, 120 (2014).

${ }^{5}$ S. J. Sweeney, Z. Batool, K. Hild, S. R. Jin, and T. J. C. Hosea, in 2011 13th International Conference On Transparent Optical Networks (ICTON), edited by Jaworski, $\mathrm{M}$ and Marciniak, M (IEEE, 2011) 13th International Conference on Transparent Optical Networks (ICTON), Stockholm, Sweden, Jun 26-30, 2011.

${ }^{6}$ Y. Bychkov and E. Rashba, JETP letters 39, 78 (1984).

${ }^{7}$ M. A. Leontiadou, K. L. Litvinenko, A. M. Gilbertson, C. R. Pidgeon, W. R. Branford, L. F. Cohen, M. Fearn, T. Ashley, M. T.
Emeny, B. N. Murdin, and S. K. Clowes, J. Phys.: Condens. Matter 23, 035801 (2011).

${ }^{8}$ G. Khodaparast, R. Meyer, X. Zhang, T. Kasturiarachchi, R. Doezema, S. Chung, N. Goel, M. Santos, and Y. Wang, Physica E 20, 386 (2004), 11th International Conference on Narrow Gap Semiconductors, Univ Buffalo, Buffalo, New York, Jun 1620, 2003.

${ }^{9}$ I. A. Kokurin, Solid State Commun. 195, 49 (2014).

${ }^{10}$ J. Heida, B. van Wees, J. Kuipers, T. Klapwijk, and G. Borghs, Phys. Rev. B 57, 11911 (1998).

${ }^{11}$ A. M. Gilbertson, W. R. Branford, M. Fearn, L. Buckle, P. D. Buckle, T. Ashley, and L. F. Cohen, Phys. Rev. B 79, 235333 (2009).

12 J. Li, A. M. Gilbertson, K. L. Litvinenko, L. F. Cohen, and S. K. Clowes, Phys. Rev. B 85, 045431 (2012).

${ }^{13}$ R. L. Kallaher and J. Heremans, Phys. Rev. B 79 (2009).

${ }^{14}$ A. M. Gilbertson, M. Fearn, J. H. Jefferson, B. N. Murdin, P. D. Buckle, and L. F. Cohen, Phys. Rev. B 77, 165335 (2008).

${ }^{15}$ K. L. Litvinenko, B. N. Murdin, J. Allam, C. R. Pidgeon, M. Bird, K. Morris, W. Branford, S. K. Clowes, L. F. Cohen, T. Ashley, and L. Buckle, New J. Phys. 8, 49 (2006).

${ }^{16}$ C. A. Broderick, M. Usman, and E. P. O'Reilly, Semicond. Sci. Technol. 28, 125025 (2013).

${ }^{17}$ C. A. Broderick, S. Mazzucato, H. Carrere, T. Amand, H. Makhlouf, A. Arnoult, C. Fontaine, O. Donmez, A. Erol, M. Usman, E. P. O'Reilly, and X. Marie, Phys. Rev. B 90, 195301 (2014).

${ }^{18}$ E. A. de Andrada e Silva, G. C. La Rocca, and F. Bassani, Phys. Rev. B 50, 8523 (1994).

${ }^{19}$ M. Usman, C. A. Broderick, Z. Batool, K. Hild, T. J. C. Hosea, S. J. Sweeney, and E. P. O'Reilly, Phys. Rev. B 87, 115104 (2013).

${ }^{20}$ K. Alberi, O. D. Dubon, W. Walukiewicz, K. M. Yu, K. Bertulis, and A. Krotkus, Appl. Phys. Lett. 91, 051909 (2007).

${ }^{21}$ P. Pfeffer and W. Zawadzki, Phys. Rev. B 59, R5312 (1999).

${ }^{22}$ I. Vurgaftman, J. R. Meyer, and L. R. Ram-Mohan, J. Appl. Phys. 89, 5815 (2001).

${ }^{23}$ B. Das, D. C. Miller, S. Datta, R. Reifenberger, W. P. Hong, P. K. Bhattacharya, J. Singh, and M. Jaffe, Phys. Rev. B 39, 1411 (1989).

${ }^{24} \mathrm{H}$. Okamoto and K. Oe, Japanese Journal of Applied Physics 38, 1022 (1999).

${ }^{25}$ D. S. Samajdar D.P., The Scientific World Journal 2014, 704830 (2014).

${ }^{26}$ R. M. Lee, J.J., Opto-Electronics Review 6, 25 (1998).

${ }^{27}$ M. K. Rajpalke, W. M. Linhart, K. M. Yu, M. Birkett, J. Alaria, J. J. Bomphrey, S. Sallis, L. F. J. Piper, T. S. Jones, M. J. Ashwin, and T. D. Veal, Appl. Phys. Lett. 105, 212101 (2014). 